\title{
Bioimpedance Analysis for Predicting Outcomes of Complex Decongestive Therapy for Gynecological Cancer Related Lymphedema: A Feasibility Study
}

\author{
Su Hwan Bae, MD, Won Jun Kim, MD, Yu Jin Seo, MD, JaYoung Kim, MD, Jae Yong Jeon, MD, PhD
}

Department of Rehabilitation Medicine, Asan Medical Center, University of Ulsan College of Medicine, Seoul, Korea

\begin{abstract}
Objective To determine whether the bioimpedance analysis (BIA) ratios of upper to lower extremities could predict treatment outcomes after complex decongestive therapy (CDT) for gynecological cancer related lymphedema (GCRL).

Methods A retrospective study, from March 2015 to December 2018, was conducted. The study sample comprised patients receiving CDT, 30 minutes per day, for 10 days. Bioimpedance was measured pre- and post-CDT. Circumference measurements were obtained at 20 and $10 \mathrm{~cm}$ above the knee (AK) and $10 \mathrm{~cm}$ below the knee (BK). We calculated the expected impedance at $0 \mathrm{~Hz}(\mathrm{R} 0)$ of extremities and upper/lower extremity R0 ratios $\left(\mathrm{R} 0_{\mathrm{U} / \mathrm{L}}\right)$. We evaluated the relationship between $\mathrm{R}_{\mathrm{U} / \mathrm{L}}$ and changes in $\mathrm{R}_{\mathrm{U} / \mathrm{L}}$ and circumferences, pre- and post-CDT.

Results Overall, 59 patients were included in this study. Thirty-one lower extremities in 26 patients comprised the acute group, and 38 lower extremities in 33 patients comprised the chronic group. Pre-treatment $\mathrm{R} 0_{\mathrm{U} / \mathrm{L}}$ was significantly correlated with $\mathrm{R}_{\mathrm{U} / \mathrm{L}}$ change after adjusting for age and $\mathrm{BMI}$ (acute: $\mathrm{R}=0.513, \mathrm{p}<0.01$; chronic: $\mathrm{R}=0.423, \mathrm{p}<0.01)$. In the acute group, pre-treatment $\mathrm{R} 0_{\mathrm{U} / \mathrm{L}}$ showed a tendency to be correlated with circumference change (AK $20 \mathrm{~cm}: \mathrm{R}=0.427, \mathrm{p}=0.02 ; \mathrm{AK} 10 \mathrm{~cm}: \mathrm{R}=0.399, \mathrm{p}=0.03$ ).

Conclusion Our study results suggested that pre-treatment BIA could predict volume reductions after CDT in the early stages of GCRL. These findings implied that BIA value could be one possible parameter to apply in treatment outcomes prediction, during the early stage of GCRL. Therefore, further large-scale prospective studies will be beneficial.
\end{abstract}

Keywords Lymphedema, Lower extremity, Gynecological cancer, Bioelectric impedance, Rehabilitation outcome

\footnotetext{
Received July 29, 2019; Revised September 3, 2019; Accepted September 20, 2019; Published online June 30, 2020

Corresponding author: Jae Yong Jeon

Department of Rehabilitation Medicine, Asan Medical Center, University of Ulsan College of Medicine, 88, Olympic-ro 43-gil, Songpa-gu, Seoul 05505, Korea. Tel: +82-2-3010-3800, Fax: +82-2-3010-6964, E-mail: jyjeon71@gmail.com

ORCID: Su Hwan Bae (https://orcid.org/0000-0003-4961-6175); Won Jun Kim (https://orcid.org/0000-0001-7087-1360); Yu Jin Seo (http://orcid. org/0000-0002-1469-1055); JaYoung Kim (https://orcid.org/0000-0002-5010-8090); Jae Yong Jeon (https://orcid.org/0000-0003-1534-7931).
}

(c) This is an open-access article distributed under the terms of the Creative Commons Attribution Non-Commercial License (http://creativecommons.org/ licenses/by-nc/4.0) which permits unrestricted noncommercial use, distribution, and reproduction in any medium, provided the original work is properly cited. Copyright ( 2020 by Korean Academy of Rehabilitation Medicine 


\section{INTRODUCTION}

Lymphedema is a chronic condition caused by dysfunction of the lymphatic system, responsible for maintaining tissue fluid balance [1]. This condition is characterized by subcutaneous accumulation of extracellular fluid, resulting from lymphatic vessel dysfunction and could lead to inflammation and fibrosis. Treatments for malignancies in cancers of the breast, uterus, ovary, and prostate are known to cause secondary lymphedema. Surgery and radiotherapy for these malignancies greatly increase the risk of lymphedema in the extremities [2]. A previous study reported that $20 \%$ of cervical cancer patients presented with lower extremity lymphedema [3]. In Koreans, $11.1 \%$ of ovarian cancer patients developed lower extremity lymphedema after treatment [4].

Lymphedema limits a patient's physical activity and increases the risk of psychosocial problems and clinical complications, including cellulitis [5]. Gynecological cancer related lymphedema (GCRL), after pelvic lymph node dissection, has been specifically shown to decrease patient quality of life [6]. Early diagnosis and treatment of lymphedema may prevent its progression and associated complications [7]. Therefore, early diagnosis and treatment are necessary for GCRL. Several different modalities are used to evaluate lymphedema, including extremity circumference measurement, volume measurement, tissue tonometry, ultrasonography, lymphoscintigraphy, dual energy X-ray absorptiometry (DEXA), and bioimpedance analysis (BIA).

Among these modalities, BIA is preferred as it is simple, inexpensive, and non-invasive. Volume measurement alone, does not account for changes in muscle mass, and is an imperfect assessment of the interstitial fluid change. Interstitial fluid change is very important in evaluating lymphedema. BIA has the advantage of being a sensitive measure of interstitial fluid changes, and a previous study has reported that multiple frequency BIA (MFBIA) reliably detects early-stage upper extremity lymphedema with a sensitivity of $100 \%$ [2]. Low frequency current usually passes through the extracellular fluid, suggesting analysis at $0 \mathrm{~Hz}$ is ideal for evaluating lymphedema [8]. However, factors such as hydration status, medical conditions, environmental factors, and ethnicity, can influence BIA results; as such, BIA absolute values alone are insufficient to discern changes of extracellular volume [9].
Therefore, a comparison of BIA values between affected and unaffected sides has been previously used to evaluate unilateral breast cancer related lymphedema (BCRL) [10].

Many studies have evaluated unilateral BCRL using BIA. BCRL presentation is usually unilateral, and BIA is, thus, performed using a ratio of affected extremity to unaffected extremity. An earlier study suggested a cutoff value of the extracellular fluid volume for assessing BCRL [11]. Another study suggested extracellular fluid volume ratio and single frequency BIA (SFBIA) before CDT, as a useful modality for predicting treatment outcomes [12].

The protocols for gynecological cancer surgery differ from those for breast cancer surgery, in that they include bilateral pelvic lymph node dissection. GCRL may thus develop on both sides, and current BIA is limited to comparisons between affected and unaffected sides. Recently Hayes et al. [13] suggested the BIA ratio of upper extremity to lower extremity for identifying cutoff values in lower extremity lymphedema in GCRL. However, research on BIA in the evaluation of GCRL remains scarce.

The purpose of this study is to investigate the feasibility of whether the BIA ratios of upper to lower extremities can reliably predict treatment outcomes for CDT in GCRL.

\section{MATERIALS AND METHODS}

\section{Study design}

This study was a retrospective study, conducted at a single lymphedema clinic, in patients who had gynecological cancer surgery from March 2015 to December 2018. All lower extremity lymphedema diagnoses were confirmed by the authors of this study. Before data was collected, approval for this study was obtained from Asan Medical Center Institutional Review Board (No. S20191328-0001).

\section{Subjects}

All patients developed lower extremity edema at least 1 month after surgery for gynecological cancer. Most patients were evaluated by D-dimer, lower extremity venography CT or Doppler ultrasonography to assess deep vein thrombosis (DVT), and lymphoscintigraphy to uncover potential secondary lymphedema before CDT. Lymphedema was diagnosed on the basis of clini- 
cal symptoms such as swelling, heaviness, tightness, and fatigue; lymphoscintigraphic findings compatible with secondary lymphedema, and at least a $2 \mathrm{~cm}$ difference in lower extremity circumference between affected and unaffected sides, regardless of subjective symptoms for unilateral lymphedema. Inclusion criteria for the study were: clinical diagnosis of unilateral or bilateral GCRL, 10-day course of CDT after the development of lymphedema, and BIA performed before commencing CDT and after the 10-day course of CDT. Exclusion criteria were the presence of comorbid conditions that could lead to lower extremity lymphedema such as current metastasis, cellulitis, DVT or incomplete medical records.

In order to identify whether BIA is a sensitive modality for early GCRL diagnosis, we then divided the patients based on the duration from symptom onset to first bioimpedance measurement, with the acute group representing duration $<6$ months and the chronic group duration $>6$ months. The duration cutoff point was chosen in accordance with a previous study [14].

\section{Lymphedema treatment}

CDT was performed on patients for 30 minutes per day for 10 days. CDT included manual lymphatic drainage, compression bandaging, skin care, and exercise education. The same physical therapist performed CDT on all patients.

\section{Lymphedema evaluation}

We conducted BIA analyses of the upper and lower extremities using the Inbody S10 (InBody, Seoul, Korea) before and after a 10-day course of CDT, as each session was conducted after a 5-minute rest. We also measured the circumferences of the lower extremities at 20 and 10 $\mathrm{cm}$ above the knee (AK) and $10 \mathrm{~cm}$ below the knee (BK), pre- and post-CDT.

In general, reactance and resistance values can be calculated using impedance and phase angle, measured at each frequency in MFBIA, and these values can be used to calculate the expected impedance measured at $0 \mathrm{~Hz}$ (R0) [15]. We calculated the R0 of the four extremities using MFBIA to evaluate GCRL in the study subjects. Previous studies have used the upper/lower extremity R0 ratio to diagnose lower extremity lymphedema [13]. Thus, we calculated the upper/lower extremity $\mathrm{R} 0$ ratio $\left(R 0_{\mathrm{U} / \mathrm{L}}\right)$, using the upper extremity $\mathrm{R} 0$ values for the ip- silateral side. We investigated the relationship between $\mathrm{R} 0_{\mathrm{U} / \mathrm{L}}$ before CDT (pre-treatment $\mathrm{R} 0_{\mathrm{U} / \mathrm{L}}$ ) of the affected side, and the changes to $\mathrm{R} 0_{\mathrm{U} / \mathrm{L}}\left(\Delta \mathrm{R} 0_{\mathrm{U} / \mathrm{L}}\right)$ and circumference values ( $\Delta$ Circumference) pre- and post-CDT, in patients with unilateral or bilateral GCRL. As the change of ratio, rather than absolute change was used as an analytic tool, all changes were presented as differences between pre- and post-CDT results.

Subgroup analysis was also performed to compare the predictive capacity of BIA between the acute and chronic groups. The primary outcomes of this study were the differences in $\mathrm{R}_{\mathrm{U} / \mathrm{L}}$ and lower extremity circumferences preand post-CDT.

\section{Statistical analyses}

All statistical analyses were conducted using SPSS version 18.0 (SPSS Inc., Chicago, IL, USA). In this study, we identified the differences between the acute and chronic groups, using an independent t-test for normally distributed data, and Mann-Whitney U-test for non-normally distributed data. The majority of data were not normally distributed, and thus we conducted Spearman correlation analysis to identify the relationships between pretreatment $\mathrm{R} 0_{\mathrm{U} / \mathrm{L}}$ of the affected side and $\Delta \mathrm{R} 0_{\mathrm{U} / \mathrm{L}}$, and $\Delta$ Circumference pre- and post-CDT. A partial correlation analysis was performed to make adjustments for age and body mass index (BMI) data that might have affected the results. A p-value of less than 0.05 was considered statistically significant.

\section{RESULTS}

\section{Subjects}

A total of 211 patients, diagnosed with GCRL and having received BIA and lower extremity circumference measurements before and after CDT, were evaluated for inclusion in this study. Of these, 152 were excluded due to meeting one or more of the exclusion criteria (BIA with measurement error, $\mathrm{n}=57$; other comorbid conditions, $\mathrm{n}=7$; incomplete medical records, $\mathrm{n}=88$ ). The remaining 59 patients were enrolled in the study. All patients were diagnosed with stage II lymphedema. The acute group comprised 31 lower extremities in 26 patients; the chronic group contained 38 lower extremities in 33 patients. The predominant gynecological cancer types were cervical cancer $(n=28)$, followed by endometrial cancer $(n=19)$, 
Table 1. Patient baseline characteristics and changes in bioimpedance values and lower extremity circumferences

\begin{tabular}{|c|c|c|c|}
\hline Clinical variable & Acute group $(n=26)$ & Chronic group $(n=33)$ & p-value \\
\hline Age (yr) & $53.38 \pm 10.23$ & $57.33 \pm 9.66$ & 0.07 \\
\hline \multicolumn{4}{|l|}{ Lesion side } \\
\hline Right & 5 & 6 & \\
\hline Left & 12 & 11 & \\
\hline Both & 9 & 16 & \\
\hline $\operatorname{BMI}\left(\mathrm{kg} / \mathrm{m}^{2}\right)$ & $23.68 \pm 2.59$ & $25.35 \pm 3.73$ & $0.03^{*}$ \\
\hline \multicolumn{4}{|l|}{ Cancer type } \\
\hline Endometrial & 8 & 11 & \\
\hline Cervical & 13 & 15 & \\
\hline Uterine & 0 & 2 & \\
\hline Ovarian & 5 & 5 & \\
\hline Lymph nodes removed & 25 & 32 & \\
\hline Radiation therapy & 13 & 14 & \\
\hline Chemotherapy & 12 & 19 & \\
\hline Lymphedema duration (mo) & $2.77 \pm 1.66$ & $48.27 \pm 54.22$ & $<0.001^{*}$ \\
\hline Pre-treatment $\mathrm{R} 0_{\mathrm{U} / \mathrm{L}}$ & $1.68(1.46-2.09)$ & $2.01(1.57-2.90)$ & 0.06 \\
\hline$\Delta \mathrm{R} 0_{\mathrm{U} / \mathrm{L}} /$ Pre-treatment $\mathrm{R} 0_{\mathrm{U} / \mathrm{L}}$ & $0.06(-0.04-0.12)$ & $-0.03(-0.11-0.05)$ & 0.06 \\
\hline$\Delta$ Circumference at $\mathrm{AK} 20 \mathrm{~cm}(\mathrm{~cm})$ & $0.70(-1.00-1.70)$ & $1.00(-0.50-2.80)$ & 0.11 \\
\hline$\Delta$ Circumference at $\mathrm{AK} 10 \mathrm{~cm}(\mathrm{~cm})$ & $0.20(-0.80-0.90)$ & $0.70(0.00-1.95)$ & $0.02^{*}$ \\
\hline$\Delta$ Circumference at $\mathrm{BK} 10 \mathrm{~cm}(\mathrm{~cm})$ & $-0.10(-0.70-0.40)$ & $0.40(-0.80-1.20)$ & 0.84 \\
\hline
\end{tabular}

Values are presented as mean \pm standard deviation or $50 \%$ quantile ( $25 \%$ quantile- $75 \%$ quantile). Thirty-one lower extremities in 26 patients comprised the acute group, and 38 lower extremities in 33 patients comprised the chronic group.

$\mathrm{BMI}$, body mass index; $\mathrm{R} 0_{\mathrm{U} / \mathrm{L}}$, upper/lower extremity impedance ratio at $0 \mathrm{~Hz}$; Pre-treatment $\mathrm{R} 0_{\mathrm{U} / \mathrm{L}}, \mathrm{R} 0_{\mathrm{U} / \mathrm{L}}$ before complex decongestive therapy; $\Delta \mathrm{R} 0_{\mathrm{U} / \mathrm{L}}, \mathrm{R} 0_{\mathrm{U} / \mathrm{L}}$ change pre- and post-treatment; $\Delta$ Circumference, circumference change preand post-treatment; $\mathrm{AK}$, above the knee; $\mathrm{BK}$, below the knee.

${ }^{*} \mathrm{p}<0.05$, statistically significant in Mann-Whitney U test.

ovarian cancer $(\mathrm{n}=10)$, and uterine cancer $(\mathrm{n}=2)$.

Table 1 shows baseline characteristics, changes in bioimpedance values, and changes in lower extremity circumferences pre- and post-CDT. No significant differences in age and bioimpedance values were observed, and only $\Delta$ Circumference at AK $10 \mathrm{~cm}$ varied significantly between the acute and chronic groups-50\% quantile (25\% quantile-75\% quantile), $0.20(-0.80-0.90)$ in the acute group vs. $0.70(0.00-1.95)$ in the chronic group; $\mathrm{p}=0.02$. BMI values in the chronic group were significantly higher than those in the acute group $\left(23.68 \pm 2.59 \mathrm{~kg} / \mathrm{m}^{2}\right.$ vs. $25.35 \pm 3.73 \mathrm{~kg} / \mathrm{m}^{2} ; \mathrm{p}=0.03$ ).

\section{Pre-treatment $R \mathrm{o}_{\mathrm{U} / \mathrm{L}}, \Delta \mathrm{Ro}_{\mathrm{U} / \mathrm{L}}$, and $\Delta$ Circumference}

Fig. 1 shows the relationships among pre-treatment $\mathrm{R} 0_{\mathrm{U} / \mathrm{L}}, \Delta \mathrm{R} 0_{\mathrm{U} / \mathrm{L}}$, and $\Delta$ Circumference in the acute and chronic groups. Pre-treatment $\mathrm{R} 0_{\mathrm{U} / \mathrm{L}}$ was significantly correlated with $\Delta \mathrm{R} 0_{\mathrm{U} / \mathrm{L}}$ pre- and post-CDT in both groups (acute: $\mathrm{R}=0.373, \mathrm{p}=0.04$ in Fig. $1 \mathrm{~A}$; chronic: $\mathrm{R}=0.354$, $\mathrm{p}=0.03$ in Fig. 1D). In the acute group, pre-treatment $\mathrm{R}_{\mathrm{U} / \mathrm{L}}$ was significantly correlated with $\Delta$ Circumference at $\mathrm{AK} 20 \mathrm{~cm}$ and $10 \mathrm{~cm}$ (AK $20 \mathrm{~cm}$ : R=0.427, p=0.02 in Fig. 1B; AK $10 \mathrm{~cm}$ : $\mathrm{R}=0.399, \mathrm{p}=0.03$ in Fig. $1 \mathrm{C}$ ). No significant correlations between pre-treatment $\mathrm{R} 0_{\mathrm{U} / \mathrm{L}}$ and $\Delta$ Circumference were observed in the chronic group; in addition, no significant differences were observed between pre-treatment $\mathrm{R} 0_{\mathrm{U} / \mathrm{L}}$ and $\Delta$ Circumference at $\mathrm{BK} 10$ $\mathrm{cm}$ in the acute group.

Table 2 shows partial correlation coefficients among pre-treatment $\mathrm{R} 0_{\mathrm{U} / \mathrm{L}}, \Delta \mathrm{R} 0_{\mathrm{U} / \mathrm{L}}$, and $\Delta$ Circumferences, after making adjustments for age and BMI. Pre-treatment $\mathrm{R} 0_{\mathrm{U} / \mathrm{L}}$ was significantly correlated with $\Delta \mathrm{R} 0_{\mathrm{U} / \mathrm{L}}$ pre- and 

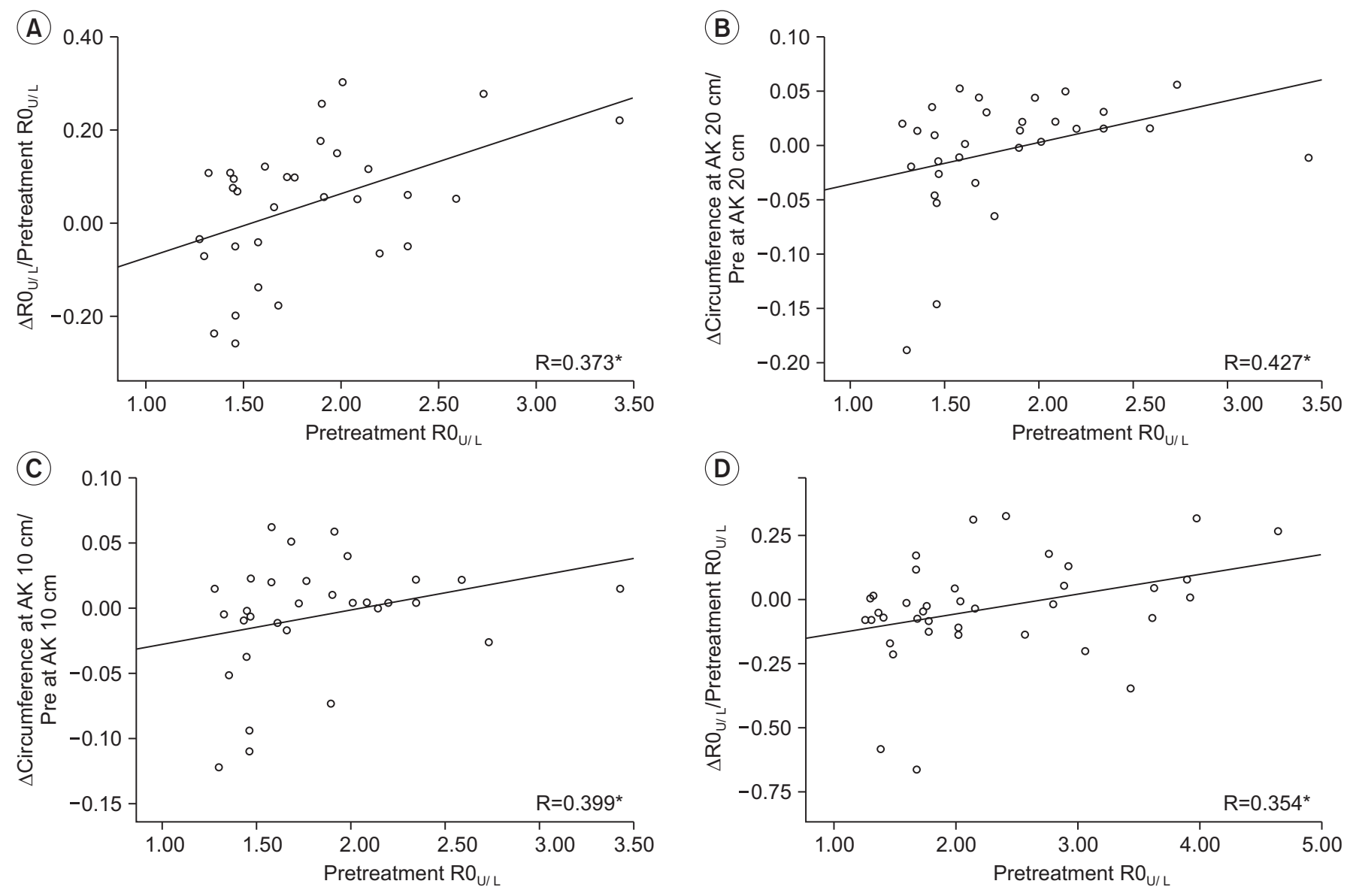

Fig. 1. Correlation of pre-treatment $\mathrm{R} 0_{\mathrm{U} / \mathrm{L}}$ and changes of lower extremity circumferences in the acute group (A, B, and C) and in the chronic group (D). $R 0_{\mathrm{U} / \mathrm{L}}$, upper/lower extremity impedance ratio at $0 \mathrm{~Hz}$; pre-treatment $\mathrm{R}_{\mathrm{U} / \mathrm{L}}, \mathrm{R} 0_{\mathrm{U} / \mathrm{L}}$ before complex decongestive therapy; $\Delta \mathrm{R} 0_{\mathrm{U} / \mathrm{L}}, \mathrm{R} 0_{\mathrm{U} / \mathrm{L}}$ change pre- and post-treatment; $\Delta$ Circumference, circumference change pre- and post-treatment; AK, Above knee; Pre, circumference before complex decongestive therapy; R, correlation coefficient in Spearman correlation analysis. ${ }^{*} p<0.05$, statistically significant in Spearman correlation analysis.

post-CDT, both in the acute and chronic group (acute: $\mathrm{R}=0.513, \mathrm{p}<0.01$; chronic: $\mathrm{R}=0.423, \mathrm{p}<0.01)$. No significant correlations were observed between pre-treatment $\mathrm{R} 0_{\mathrm{U} / \mathrm{L}}$ and $\Delta$ Circumferences after adjusting for age and BMI. Although not statistically significant, in the acute group, pre-treatment $\mathrm{R} 0_{\mathrm{U} / \mathrm{L}}$ tended to be positively correlated with $\Delta$ Circumference.

\section{DISCUSSION}

Our results suggest that pre-treatment BIA can predict lower extremity circumference changes, reflecting volume changes after CDT, in the early stages of GCRL. To the best of our knowledge, this is the first study to clarify the relationship between pre-treatment BIA and volume change after CDT in the GCRL.
In this study, lymphedema was improved after CDT in most patients. However, some patients had no lymphedema improvement or got worse after CDT. CDT was performed on an outpatient basis, and the effects of CDT may have been different because of variability in patient compliance. This study investigated whether pre- and post-treatment BIA results reflect lymphedema changes pre- and post-CDT, rather than using BIA itself to diagnose lymphedema.

Previous studies have set specific diagnostic criteria for lower extremity lymphedema. In this study, the ratio of upper extremity R0 to lower extremity R0 was applied in such a way as to match against a cutoff value, used to diagnose lower extremity lymphedema. These values were $>1.308$ on the dominant side, and $>1.340$ on the nondominant side (sensitivity $75 \%$, specificity $85 \%$ ), as deter- 
Table 2. Partial correlation coefficients between pre-treatment $\mathrm{R} 0_{\mathrm{U} / \mathrm{L}}$ and changes to lower extremity circumferences after making adjustments for age and BMI

\begin{tabular}{|c|c|c|c|}
\hline & & Pre-treatment $\mathrm{RO}_{\mathrm{U} / \mathrm{L}}$ & p-value \\
\hline \multirow[t]{4}{*}{ Acute group $(\mathrm{n}=31)$} & $\Delta \mathrm{R} 0_{\mathrm{U} / \mathrm{L}} /$ Pre-treatment $\mathrm{R} 0_{\mathrm{U} / \mathrm{L}}$ & 0.513 & $0.004^{*}$ \\
\hline & $\Delta$ Circumference / Pre at AK $20 \mathrm{~cm}$ & 0.357 & 0.06 \\
\hline & $\Delta$ Circumference / Pre at AK $10 \mathrm{~cm}$ & 0.307 & 0.10 \\
\hline & $\Delta$ Circumference / Pre at BK $10 \mathrm{~cm}$ & 0.259 & 0.18 \\
\hline \multirow[t]{4}{*}{ Chronic group $(\mathrm{n}=38)$} & $\Delta \mathrm{R} 0_{\mathrm{U} / \mathrm{L}} /$ Pre-treatment $\mathrm{R} 0_{\mathrm{U} / \mathrm{L}}$ & 0.423 & $0.01^{*}$ \\
\hline & $\Delta$ Circumference / Pre at AK $20 \mathrm{~cm}$ & -0.016 & 0.93 \\
\hline & $\Delta$ Circumference / Pre at AK $10 \mathrm{~cm}$ & 0.254 & 0.14 \\
\hline & $\Delta$ Circumference / Pre at BK $10 \mathrm{~cm}$ & -0.190 & 0.27 \\
\hline
\end{tabular}

Values are presented as partial correlation coefficient $(\mathrm{R})$ between two parameters.

$\mathrm{BMI}$, body mass index; $\mathrm{R}_{\mathrm{U} / \mathrm{L}}$, upper/lower extremity impedance ratio at $0 \mathrm{~Hz}$; Pre-treatment $\mathrm{R} 0_{\mathrm{U} / \mathrm{L}}, \mathrm{R}_{\mathrm{U} / \mathrm{L}}$ before complex decongestive therapy; $\Delta \mathrm{R} 0_{\mathrm{U} / \mathrm{L}}, \mathrm{R} 0_{\mathrm{U} / \mathrm{L}}$ change before and after treatment; $\Delta$ Circumference, circumference change before and after treatment; Pre, circumference before complex decongestive therapy; AK, above knee; BK, below knee. ${ }^{*} \mathrm{p}<0.05$, statistically significant in partial correlation analysis.

mined by a previous study [13]. Other studies have also used these cutoff values to diagnose GCRL [16]. Another study reported, that BIA was an appropriate method for the assessment of extracellular fluid in the lower extremities of healthy young people [17]. Most studies, however, have focused on the diagnostic validity of BIA for lower extremity lymphedema.

In BCRL, a previous study evaluated the calculated extracellular fluid ratio of affected versus unaffected upper extremities with BIA and changes to upper extremity circumferences pre- and post-CDT. The result of that study suggested that pretreatment BIA values could predict treatment outcomes of CDT [12].

In our study, we verified that pre-treatment BIA values could predict the treatment outcomes of CDT in GCRL. $\mathrm{R} 0_{\mathrm{U} / \mathrm{L}}$ increased as lower extremity lymphedema worsened. As such, an increase in $\Delta \mathrm{R}_{\mathrm{U} / \mathrm{L}}$ pre- to post-CDT, indicated lymphedema improvement. Pre-treatment $\mathrm{R} 0_{\mathrm{U} / \mathrm{L}}$ was positively correlated with $\Delta \mathrm{R} 0_{\mathrm{U} / \mathrm{L}}$ in both the acute and chronic groups, suggesting that pre-treatment $\mathrm{RO}_{\mathrm{U} / \mathrm{L}}$ was a reliable factor for predicting changes after CDT in GCRL. Furthermore, pre-treatment $\mathrm{R}_{\mathrm{U} / \mathrm{L}}$ showed a tendency to be correlated with $\Delta$ Circumference in the acute group, although this correlation was not statistically significant after adjusting for age and BMI. These findings suggested, that lower extremity circumferences were further reduced after CDT, in patients with severe lymphedema in the acute group. These results indicated that pre-treatment $\mathrm{R} 0_{\mathrm{U} / \mathrm{L}}$ held potential for predicting treatment outcomes for GCRL in the acute patients' group. Therefore, we concluded that BIA predicted more reliably the effects of CDT for GCRL in the early stages of lymphedema, as opposed to the chronic stages. BIA might reflect accurately the extracellular fluid volume by calculating electric current flow through the body and is based on the principle that fat impedes electric current more than protein or fluid [9]. In the chronic GCRL group (lymphedema duration $\geq 6$ months), chronic inflammation and fibrosis may promote fat hypertrophy [1], that could influence the BIA results in these patients. A lack of previous studies on the effects of different periods of duration of lymphedema on BIA results, limits the applicability of dividing the acute and chronic groups using a 6-month cutoff. Future studies, evaluating differences in BIA results according to lymphedema duration, will be necessary to explore this phenomenon in more depth.

Several limitations of this study must be acknowledged. First, the sample size was small and heterogeneous regarding cancer type, stage, and treatment. The correlations between pre-treatment $\mathrm{R} 0_{\mathrm{U} / \mathrm{L}}$ and $\Delta$ Circumference were not statistically significant, after adjusting for age and BMI (a potential effect of the small sample size). Further studies with larger populations were recommended. Second, while $\mathrm{R} 0_{\mathrm{U} / \mathrm{L}}$ represented the overall impedance measurement of the lower extremity, $\Delta$ Circumference might be less statistically significant because it only assessed changes to specific segments of the lower extrem- 
ity. Further large-scale studies evaluating BIA for the segmental area of the lower extremity will be necessary. Third, unlike previous studies, the dominant extremity was not identified in this medical record review, preventing us from distinguishing between dominant and nondominant extremities, when calculating $\mathrm{R} 0_{\mathrm{U} / \mathrm{L}}$. Finally, we could not control factors potentially affecting the results of BIA, such as temperature, hydration status, and physical activity just before measurement, all of which might have affected our results. While further studies are recommended, this study confirms the feasibility of BIA for predicting treatment outcomes of CDT in the early stages of GCRL.

In conclusion, findings from this study suggested that pre-treatment BIA values can predict interstitial fluid volume reductions after CDT in the early stages of GCRL. These findings implied that BIA parameters could be used to predict the treatment outcomes in the early stage of GCRL. We recommend further large-scale prospective studies to be conducted for more in-depth research.

\section{CONFLICT OF INTEREST}

No potential conflict of interest relevant to this article was reported.

\section{ACKNOWLEDGMENTS}

This work was supported by the National Research Foundation of Korea (NRF) grant funded by the Korea government (MSIT) (No. 2019R1A2C100905512).

\section{AUTHORS CONTRIBUTION}

Conceptualization: Bae SH, Jeon JY. Methodology: Bae SH, Kim WJ, Kim JY, Jeon JY, Seo YJ. Formal analysis: Bae SH, Jeon JY. Project administration: Bae SH, Jeon JY, Kim JY, Kim WJ, Seo YJ. Visualization: Bae SH, Jeon JY, Kim JY. Writing - original draft: Bae SH, Kim JY, Jeon JY. Writing review and editing: Bae SH, Jeon JY, Kim JY, Kim WJ, Seo YJ. Approval of final manuscript: all authors.

\section{REFERENCES}

1. Mortimer PS, Rockson SG. New developments in clinical aspects of lymphatic disease. J Clin Invest
2014;124:915-21.

2. Cornish BH, Chapman M, Hirst C, Mirolo B, Bunce $\mathrm{IH}$, Ward LC, et al. Early diagnosis of lymphedema using multiple frequency bioimpedance. Lymphology

3. 2001;34:2-11.

4. Ohba Y, Todo Y, Kobayashi N, Kaneuchi M, Watari H, Takeda M, et al. Risk factors for lower-limb lymphedema after surgery for cervical cancer. Int J Clin Oncol 2011;16:238-43.

5. Ki EY, Park JS, Lee KH, Hur SY. Incidence and risk factors of lower extremity lymphedema after gynecologic surgery in ovarian cancer. Int J Gynecol Cancer 2016;26:1327-32.

6. Schmitz KH. Balancing lymphedema risk: exercise versus deconditioning for breast cancer survivors. Exerc Sport Sci Rev 2010;38:17-24.

7. Kim SI, Lim MC, Lee JS, Lee Y, Park K, Joo J, et al. Impact of lower limb lymphedema on quality of life in gynecologic cancer survivors after pelvic lymph node dissection. Eur J Obstet Gynecol Reprod Biol 2015;192:31-6.

8. Polat AK, Karabacak U, Mutlu V, Tomak L, Bilgici A. Early diagnosis of lymphedema after breast cancer treatment: bio-impedance spectroscopy. J Breast Health 2017;13:83-7.

9. Gaw R, Box R, Cornish B. Bioimpedance in the assessment of unilateral lymphedema of a limb: the optimal frequency. Lymphat Res Biol 2011;9:93-9.

10. Dehghan M, Merchant AT. Is bioelectrical impedance accurate for use in large epidemiological studies? Nutr J 2008;7:26.

11. Ward LC. Bioelectrical impedance analysis: proven utility in lymphedema risk assessment and therapeutic monitoring. Lymphat Res Biol 2006;4:51-6.

12. Jung M, Jeon JY, Yun GJ, Yang S, Kwon S, Seo YJ. Reference values of bioelectrical impedance analysis for detecting breast cancer-related lymphedema. Medicine (Baltimore) 2018;97:e12945

13. Kim L, Jeon JY, Sung IY, Jeong SY, Do JH, Kim HJ. Prediction of treatment outcome with bioimpedance measurements in breast cancer related lymphedema patients. Ann Rehabil Med 2011;35:687-93.

14. Hayes SC, Janda M, Steele M, Cornish B, Ward L, Box $\mathrm{R}$, et al. Identifying diagnostic criteria for upperand lower-limb lymphoedema. Brisbane, Australia; Queensland University of Technology; 2016. 
15. Norman SA, Localio AR, Potashnik SL, Simoes Torpey HA, Kallan MJ, Weber AL, et al. Lymphedema in breast cancer survivors: incidence, degree, time course, treatment, and symptoms. J Clin Oncol 2009;27:390-7.

16. Stroud DB, Cornish BH, Thomas BJ, Ward LC. The use of Cole-Cole plots to compare two multifrequency bioimpedance instruments. Clin Nutr 1995;14:307-11.

17. Hayes SC, Janda M, Ward LC, Reul-Hirche H, Steele $\mathrm{ML}$, Carter J, et al. Lymphedema following gynecolog- ical cancer: results from a prospective, longitudinal cohort study on prevalence, incidence and risk factors. Gynecol Oncol 2017;146:623-9.

18. Douglass J, Graves P, Gordon S. Intrarater reliability of tonometry and bioimpedance spectroscopy to measure tissue compressibility and extracellular fluid in the legs of healthy young people in Australia and Myanmar. Lymphat Res Biol 2017;15:57-63. 